\title{
Variation in male fertility explains an apparent effect of genotypic diversity on success in larval competition in Drosophila melanogaster
}

\author{
Kevin Fowler and \\ Linda Partridge
}

Department of Zoology, University of Edinburgh, West Mains Road, Edinburgh, EH9 3JT, Scotland.

Other workers have reported an effect of genotypic diversity on larval viability in Drosophila melanogaster. We have found that in one case at least, the impaired larval viability of a mixture of half-sibs compared to unrelated larvae is attributable to an effect of male fertility on progeny density and not to an effect of relatedness on progeny survival.

\section{INTRODUCTION}

Several hypotheses for the maintenance of genetic polymorphism and sexual reproduction suggest that individuals of the same genotype may compete more intensely than less similar morphs (e.g., Levene, 1953; Maynard Smith, 1971; Williams, 1975; Bell, 1982). One line of evidence in support of this point of view comes from experiments where a mixture of competing genotypes is demonstrated to be more productive than a monoculture (e.g., Battaglia and Smith, 1961; Kearsey, 1965; Ellstrand and Antonovics, 1985).

A recent experiment of this kind manipulated genetic diversity by altering the degree of relatedness between competing larvae of an outbred wild type strain of Drosophila melanogaster (PérezTomé and Toro, 1982). Vials with eggs laid by 10 unrelated females each mated to a different male (heterogeneous series) produced more surviving adults than did vials with eggs laid by 10 unrelated females each mated to the same male (homogeneous series). Vials in the heterogeneous series contained larvae of a mixture of 10 fullsibships whereas those in the homogeneous series contained larvae of 10 full sib-ships each related as half-sibs through a common father. The genotypic diversity should therefore be lower in the homogeneous series, and Pérez-Tomé and Toro (1982) interpreted their results as evidence for frequency-dependent selection arising either by differential resource utilisation by different genotypes or by some other genotype-specific interaction between larvae. This interpretation was queried by Fowler and Seger (1983) who suggested an alternative explanation based on directional selection for an unconditionally favourable allele A affecting larval survival, and a law of diminishing returns whereby vials with high frequencies of $A$ had lower per capita survival rates than vials with low frequencies.

A third interpretation of the result is also possible, in terms of variation in male fertility. Several studies have demonstrated the importance of variation in male fertility in Drosophila (e.g., Kvelland, 1965; Brittnacher, 1981; Partridge et al., in press; Maynard Smith, 1956; Steele, 1984). In PérezTomé and Toro's experiment each female in both series was one of a mating group of 10 inseminated by the same male. In the homogeneous series, these mating groups were kept together and put to lay in a single vial whereas in the heterogeneous series groups of 10 laying females were constructed by taking each female from a different mating group. If male fertility was variable, the between-vial variance (but not the mean) of the number of fertile eggs produced would have been higher in the homogeneous series, because only one male contributed mates to each vial, whereas 10 did so in the heterogeneous series. This difference in variance was found but not commented on by Pérez Tomé and Toro (see Results), and suggests that standardisation of the number of laying females may not have resulted in standardisation of the number of eggs laid. If, as a result of larval competition, survival rates declined with increasing larval 
density as has been previously demonstrated (e.g., Kearsey, 1965; Bos and Scharloo, 1974), the results of Pérez Tomé and Toro could be explained; the higher variance in the homogeneous series could have resulted in a higher proportion of larvae being present in higher-than-average-density vials with associated low survival rates and hence a lower total productivity of adults for the series. If this interpretation is correct, it is the higher variance in fertile egg production in the homogeneous series that is critical rather than lower success in competition of the hatching larvae, and standardisation of larval density should abolish the difference between the two series.

The aim of the present work was to replicate Pérez-Tomé and Toro's results and to investigate their cause, by examining rates of fertile egg production, productivity of vials set up with different densities of larvae and the effects of standardisation of larval density on the results.

\section{MATERIALS AND METHODS}

We used an outbred wild-type stock collected in Brighton by Dr G. S. Wilkinson in 1984 and maintained since then in population cages on Edinburgh food medium made by adding $20 \mathrm{~g}$ agar, $22 \mathrm{~g}$ flaked yeast, $150 \mathrm{~g}$ maize meal, $130 \mathrm{~g}$ treacle, $5 \mathrm{~g}$ nipagin and $5 \mathrm{ml}$ propionic acid to 1.3 litres of water. The flies were kept at $25 \pm 1 \cdot 5^{\circ} \mathrm{C}$ with a fixed illumination cycle of 12 hours dark followed by 12 hours light. All handling was performed at room temperature using carbon dioxide anaesthesia.

\section{Experiment 1 Replication of Pérez-Tomé and Toro's experimental design}

Virgin males and females were collected and aged for 3 days. 76 mating groups of 1 male and 10 females were set up in vials and stored for 4 days, after which the male was discarded. Two types of laying group were then produced. Firstly, sets of 10 females from the same mating group were placed in $75 \times 24 \mathrm{~mm}$ food-vials containing $7 \mathrm{ml}$ of medium to form the homogeneous series of vials. Secondly, sets of 10 females randomly taken from 10 different mating groups were placed in food-vials to form the heterogeneous series of vials. In both series, each set of females was transferred to fresh vials every 24 hours until four vials had been obtained. Subsequently, we obtained daily progeny counts for each vial until the cultures were exhausted.

\section{Experiment 2 Reduction of the amount of food}

In view of the results from experiment 1 , we wished to increase the level of larval competition. Accordingly, we repeated the procedure of experiment 1 with 68 mating groups and reduced the amount of food available to the larvae by 75 per cent by using $50 \times 12 \mathrm{~mm}$ food-vials containing $1.75 \mathrm{ml}$ of medium. The vials used by Pérez-Tomé and Toro (1982) were $90 \times 30 \mathrm{~mm}$ containing $2 \mathrm{ml}$ of a different (Lewis) medium.

\section{Experiment 3 Examination of progeny production and the effect of standardisation of larval density}

The male fertility explanation predicts that the variance in fertile egg production should be greater in the homogeneous series. We tested this prediction directly by counting the number of early larvae produced by groups of laying females of the two series. The mating scheme for these females was the same as that of experiments 1 and 2 . In order to make counting both straightforward and accurate, each set of inseminated females was allowed to lay eggs on the surface of medium in a $2 \mathrm{ml}$ plastic spoon. The medium was made by adding $300 \mathrm{ml}$ of sweetened grape-juice concentrate and $40 \mathrm{~g}$ agar to $300 \mathrm{ml}$ of water and allowed to set in the spoons after which its surface was painted with a yeast solution. The females were allowed to lay eggs for 24 hours. After 36 hours, we counted the number of larvae on each spoon. This procedure was performed on two successive days and yielded total sample sizes of 78 for the homogeneous series and 65 for the heterogeneous series.

We also examined the effect of standardisation of larval density on the results by setting up vials with an initial larval density of 100 larvae per vial. If the male fertility explanation is correct, then there ought to be no significant difference in productivity between series. Using paintbrushes, we transferred the larvae from the surface of the spoons to a small food-vial of the same size as those used in experiment 2. This was done on two consecutive days. The procedure was laborious and so it was only possible to attain total sample sizes of 49 and 48 for the homogeneous and heterogeneous series respectively. We made daily progeny counts for each vial until the cultures were exhausted.

\section{Experiment 4 Range of larval densities}

To see whether larval survival declined with increasing larval densities, we set up 26 vials with 
a wide range of initial larval densities (from 45 to 252 larvae per vial). This range covers the range of densities measured in experiment 3 . We used the same method as in experiment 3 to produce larvae for transfer to small food-vials of the same size as those used in experiment 2 and made daily progeny counts on each vial until the cultures were exhausted. All of the vials were set up using progeny from flies of the homogeneous series in experiment 3 .

\section{RESULTS}

For comparison with the results of our experiments, we have summarised Pérez-Tomé and Toro's results in table 1 . This shows the mean productivity for each day-long egg-laying period and the total productivity for each series together with the results of the t-tests between series' means. For each period, the mean productivity of the heterogeneous series exceeded that of the homogeneous series although this difference was significant in period 2 only $(p<0.001)$. The difference between the overall mean productivity of the series was highly significant $(p<0.005)$. Pérez-Tomé and Toro did not comment on the pattern of the variance in productivity between vials within series. We have calculated approximate $F$-values based upon their published standard errors and these are included in table 1 together with their associated probabilities. The $F$-values are based on Pérez-Tomé and Toro's description of the sample size as approximately 35 . For each period, the variance of the productivity was greater in the homogeneous series. This difference was almost significant in period $2(p=0.06)$ and highly significant for period $3(p=0.01)$, and the difference in variance in total productivity was highly significant $(p<0.001)$.

\section{Experiment 1 Replication of Pérez-Tomé and Toro's experimental design}

The results are given in table 2 . There were only small differences between the two series in mean productivity. These were significant in only one of the four subsets (period $3, p<0.05$ ), and the overall mean productivity of the two series was almost identical $(p=0.96)$. The pattern of $F$-values showed that the variance of the homogeneous series was always the larger and the difference was significant in three of the subsets (period 1, $p<$ 0.001 ; period $2, p<0.01$; period $4, p<0.05$ ). The variance of the overall productivity was also greater in the homogeneous series $(p<0.001)$.

\section{Experiment 2 Reduction of the amount of food}

The results are given in table 3 . The variance in

Table 1 Summary of the results of Pérez-Tomé and Toro (1982). The mean number of adults emerging per vial for the different egg-laying periods of each experimental series (values shown as mean \pm s.e.). Column 3 gives value of $t$ statistic with its associated probability for the observed difference in means. Column 4 gives value of $F$ statistic with its associated probability for the observed ratio of variances.

\begin{tabular}{|c|c|c|c|c|c|c|}
\hline & $\begin{array}{l}\text { Heterogeneous } \\
\text { series }\end{array}$ & $\begin{array}{l}\text { Homogeneous } \\
\text { series }\end{array}$ & $t$ & $p$ & $F$ & $p$ \\
\hline Period 1 & $58 \cdot 46 \pm 3 \cdot 16$ & $51 \cdot 88 \pm 3 \cdot 41$ & 1.43 & $<0 \cdot 20$ & $1 \cdot 16$ & 0.66 \\
\hline Period 2 & $53 \cdot 62 \pm 2 \cdot 41$ & $39 \cdot 88 \pm 3 \cdot 33$ & $3 \cdot 38$ & $<0.001$ & 1.91 & $0 \cdot 06$ \\
\hline Period 3 & $42 \cdot 62 \pm 1 \cdot 93$ & $38 \cdot 51 \pm 3 \cdot 00$ & $1 \cdot 16$ & $<0.30$ & $2 \cdot 42$ & 0.01 \\
\hline Period 4 & $37 \cdot 49 \pm 2 \cdot 04$ & $32 \cdot 86 \pm 2 \cdot 35$ & 1.49 & $<0 \cdot 20$ & $1 \cdot 33$ & 0.41 \\
\hline Total & $192 \cdot 20 \pm 5 \cdot 3$ & $159 \cdot 69 \pm 9 \cdot 90$ & $2 \cdot 94$ & $<0.005$ & $3 \cdot 49$ & $<0.001$ \\
\hline
\end{tabular}

Table 2 The mean number of adults emerging per vial for the different egg-laying periods of each experimental series (values shown as mean \pm s.e.). Column 3 gives the value of the $t$ statistic with its associated probability for the observed difference in means. Column 4 gives the value of the $F$ statistic with its associated probability for the observed ratio of variances.

\begin{tabular}{|c|c|c|c|c|c|c|}
\hline & $\begin{array}{l}\text { Heterogeneous } \\
\text { series }\end{array}$ & $\begin{array}{l}\text { Homogeneous } \\
\text { series }\end{array}$ & $t$ & $p$ & $F$ & $p$ \\
\hline Period 1 & $56 \cdot 08 \pm 2 \cdot 55$ & $58 \cdot 84 \pm 5 \cdot 47$ & $0 \cdot 46$ & 0.647 & $4 \cdot 6$ & $<0.001$ \\
\hline Period 2 & $32 \cdot 89 \pm 1 \cdot 66$ & $36 \cdot 37 \pm 2 \cdot 54$ & $1 \cdot 15$ & $0 \cdot 254$ & $2 \cdot 36$ & 0.01 \\
\hline Period 3 & $23 \cdot 39 \pm 1 \cdot 32$ & $19 \cdot 16 \pm 1 \cdot 55$ & $2 \cdot 08$ & $<0.05$ & $1 \cdot 37$ & 0.40 \\
\hline Period 4 & $33 \cdot 11 \pm 1 \cdot 34$ & $30 \cdot 79 \pm 1.95$ & 0.98 & $0 \cdot 33$ & $2 \cdot 11$ & $<0.05$ \\
\hline Total & $145 \cdot 63 \pm 4 \cdot 39$ & $145 \cdot 16 \pm 9 \cdot 28$ & 0.05 & 0.96 & $4 \cdot 46$ & $<0.001$ \\
\hline
\end{tabular}


productivity of the homogeneous series was the larger in every case and was significantly so for two of the subsets (period 1, $p<0.005$; period 4, $p<0.005)$ and for the variance of overall productivity $(p<0.003)$. This pattern of $F$-values was broadly similar to that of experiment 1 . The mean productivity of the heterogeneous series always exceeded that of the homogeneous series. This difference was highly significant for period 1 ( $p<$ $0.005)$; period $3(p<0.001)$ and the overall mean productivity $(p<0.005)$. These differences in mean productivity between series were considerably greater than those found in experiment 1 and resemble those reported by Pérez-Tomé and Toro (1982).

\section{Experiment 3 Examination of progeny production, and the effect of standardisation of larval density}

Table 4 summarises the results of the larval counts for laying groups of females of each series. On both days the variance in larval density between vials of the homogeneous series was greater than that for the heterogeneous series. This difference was highly significant in both cases (day $1, F=3.61$ $p<0.001$; day $2, F=3.41 p<0.002$ ). The mean larval densities of the two series did not differ significantly on either day (day $1, t=1.41 p=$ 0.163 ; day $2, t=0.81 p=0.421$ ). Table 5 gives the productivities of the vials of standard initial larval density. On both days, the mean productivity of the standard density vials in the homogeneous series was higher than that for vials in the heterogeneous series. However, this difference was not significant in either case (day $1, t=1.03 p=$ 0.309 ; day $2, t=1.57 p=0.123$ ). On day 1 , the variance of the productivity of the homogeneous series was larger than that of the heterogeneous series but not significantly so $(F=1 \cdot 69, p=0 \cdot 22)$. On day 2 , the variance difference was reversed but this difference was also not significant $(F=1 \cdot 58$, $p=0 \cdot 32$ ).

\section{Experiment 4 Range of larval densities}

The results are shown in fig. 1 . The productivity of each of the 26 vials (expressed as a percentage of the initial larval density) was plotted against the initial larval density. It is apparent that there is a decline in productivity with increasing larval density. This negative correlation is highly sig-

Table 3 Mean number of adults emerging per vial for the different egg-laying periods of each experimental series (values shown as mean \pm s.e.). Column 3 gives the value of the $t$ statistic with its associated probability for the observed difference in means. Column 4 gives the value of the $F$ statistic with its associated probability for the observed ratio of variances

\begin{tabular}{|c|c|c|c|c|c|c|}
\hline & $\begin{array}{l}\text { Heterogeneous } \\
\text { series }\end{array}$ & $\begin{array}{l}\text { Homogeneous } \\
\text { series }\end{array}$ & $t$ & $p$ & $F$ & $p$ \\
\hline Period 1 & $74 \cdot 62 \pm 3 \cdot 07$ & $55 \cdot 74 \pm 5 \cdot 31$ & 3.08 & $<0.005$ & $3 \cdot 00$ & $<0.005$ \\
\hline Period 2 & $40 \cdot 53 \pm 2 \cdot 85$ & $31 \cdot 91 \pm 3 \cdot 53$ & 1.90 & 0.062 & 1.54 & 0.26 \\
\hline Period 3 & $35.65 \pm 1.73$ & $24.03 \pm 1.99$ & 4.4 & $<0.001$ & $1 \cdot 33$ & 0.48 \\
\hline Period 4 & $31 \cdot 50 \pm 1 \cdot 45$ & $27 \cdot 76 \pm 2 \cdot 45$ & $1 \cdot 31$ & $0 \cdot 195$ & $2 \cdot 88$ & $<0.005$ \\
\hline Total & $180 \cdot 41 \pm 6.44$ & $139 \cdot 44 \pm 11 \cdot 22$ & $3 \cdot 17$ & $<0.005$ & 3.03 & $<0.003$ \\
\hline
\end{tabular}

Table 4 Mean number of larvae counted for sets of females of each experimental series. Column 3 gives the value of the $t$ statistic with its associated probability for the observed difference in means. Column 4 gives the value of the $F$ statistic with its associated probability for the observed ratio of variances

\begin{tabular}{|c|c|c|c|c|c|c|}
\hline & $\begin{array}{l}\text { Heterogeneous } \\
\text { series }\end{array}$ & $\begin{array}{l}\text { Homogeneous } \\
\text { series }\end{array}$ & $t$ & $p$ & $F$ & $p$ \\
\hline \multicolumn{7}{|l|}{ Day 1} \\
\hline Number of vials & 36 & 44 & & & & \\
\hline Mean larval count & $113 \cdot 78$ & $124 \cdot 82$ & $1 \cdot 41$ & $0 \cdot 163$ & 3.61 & $<0.001$ \\
\hline Variance of larval count & $555 \cdot 28$ & $2005 \cdot 74$ & & & & \\
\hline \multicolumn{7}{|l|}{ Day 2} \\
\hline Number of vials & 29 & 34 & & & & \\
\hline Mean larval count & $165 \cdot 00$ & $152 \cdot 50$ & 0.81 & 0.421 & $3 \cdot 41$ & $<0.002$ \\
\hline Variance of larval count & $1754 \cdot 28$ & $5984 \cdot 84$ & & & & \\
\hline
\end{tabular}


Table 5 The mean number of adults emerging per vial of each experimental series from vials with an initial larval density of 100 . Column 3 gives the value of the $t$ statistic with its associated probability for the observed difference in means. Column 4 gives the value of the $F$ statistic with its associated probability for the observed ratio of variances.

\begin{tabular}{|c|c|c|c|c|c|c|}
\hline & $\begin{array}{l}\text { Heterogeneous } \\
\text { series }\end{array}$ & $\begin{array}{l}\text { Homogeneous } \\
\text { series }\end{array}$ & $t$ & $p$ & $F$ & $p$ \\
\hline \multicolumn{7}{|l|}{ Day 1} \\
\hline Number of vials & 23 & 24 & & & & \\
\hline Mean productivity & $75 \cdot 43$ & $79 \cdot 5$ & 1.03 & $0 \cdot 309$ & 1.69 & $0 \cdot 22$ \\
\hline Variance of productivity & $135 \cdot 81$ & $229 \cdot 92$ & & & & \\
\hline \multicolumn{7}{|l|}{ Day 2} \\
\hline Number of vials & 25 & 25 & & & & \\
\hline Mean productivity & $79 \cdot 32$ & $82 \cdot 8$ & $1 \cdot 57$ & $0 \cdot 123$ & $1 \cdot 58$ & $0 \cdot 32$ \\
\hline Variance of productivity & $74 \cdot 54$ & $47 \cdot 2$ & & & & \\
\hline
\end{tabular}

nificant $(r=-0.88, \quad p<0.001)$. A curvilinear regression analysis indicated that the data could be best explained by a straight line of the form:

$$
y=110 \cdot 57-0 \cdot 277 . x .
$$

As a consequence of this linear decline in productivity, the number of adults is a convex function of initial larval density.

It would be interesting to use the fitted line/curve to estimate the adult productivity for each of the larval counts found in experiment 3. The mean and variance of the estimated values could be compared with that of the productivities observed for each series in experiment 2. However, this type of comparison between experiments is inappropriate because of the differences in environmental conditions. In experiment 3 the surface of the medium on the spoons was painted with a yeast solution (see Materials and Methods), whereas unyeasted food-vials were used in experiment 2. Consequently, the inseminated females were well-fed in experiment 3 and may have laid a higher number of fertile eggs than in experiment 2 . The yeast solution will also have provided nutrition in early larval life for larvae on the spoons, so that those larvae could have experienced a lower level of mortality subsequently than those in experiment 2 .

\section{DISCUSSION}

The results support the idea that the findings of Pérez-Tomé and Toro can be explained entirely in terms of variation in male fertility. The higher variance in fertile egg production by the homogeneous series in Experiment 3 supports this explanation; variation in female fertility, although undoubtedly present, should not have differed between the females in the two series. The fertility explanation is also supported by the finding that the difference in adult productivity between the series was abolished by standardisation of larval density, suggesting that the difference was not a consequence of a difference in success in larval competition. Larval density was standardised at a level slightly below that of the mean larval density found on day 1 in experiment 3 , so that any difference in competitive success should have been apparent. The results of manipulating larval density support the idea that the reduced adult productivity in the homogeneous series was instead a consequence of lower per capita survival rates in the higher density vials. The difference in variance in density between the series did not always produce a difference in mean adult productivity and whether or not it did so appeared to depend upon the level of larval competition, since a difference in mean adult productivity was found at the higher larval densities in experiment 2 but not in experiment 1 .

It is clearly therefore not appropriate to assume that standardisation of adult density will result in standard densities of progeny, and the present results suggest that variation in male as well as female fertility can be important in producing variation in rates of progeny production. To test for frequency-dependent or other interactions between competing larvae it is therefore essential to standardise larval density directly, as has been demonstrated in a different context by Caligari and Baban (1981), who showed that a decline in fertility with age accounted for an apparent effect of parental age on heritability of bristle number.

Our results do not demonstrate that genotype diversity is without any effect on larval survival rates even in the stock used. They simply show that the difference in genotype diversity between the series was not sufficient to produce a measurable effect with our sample sizes. 


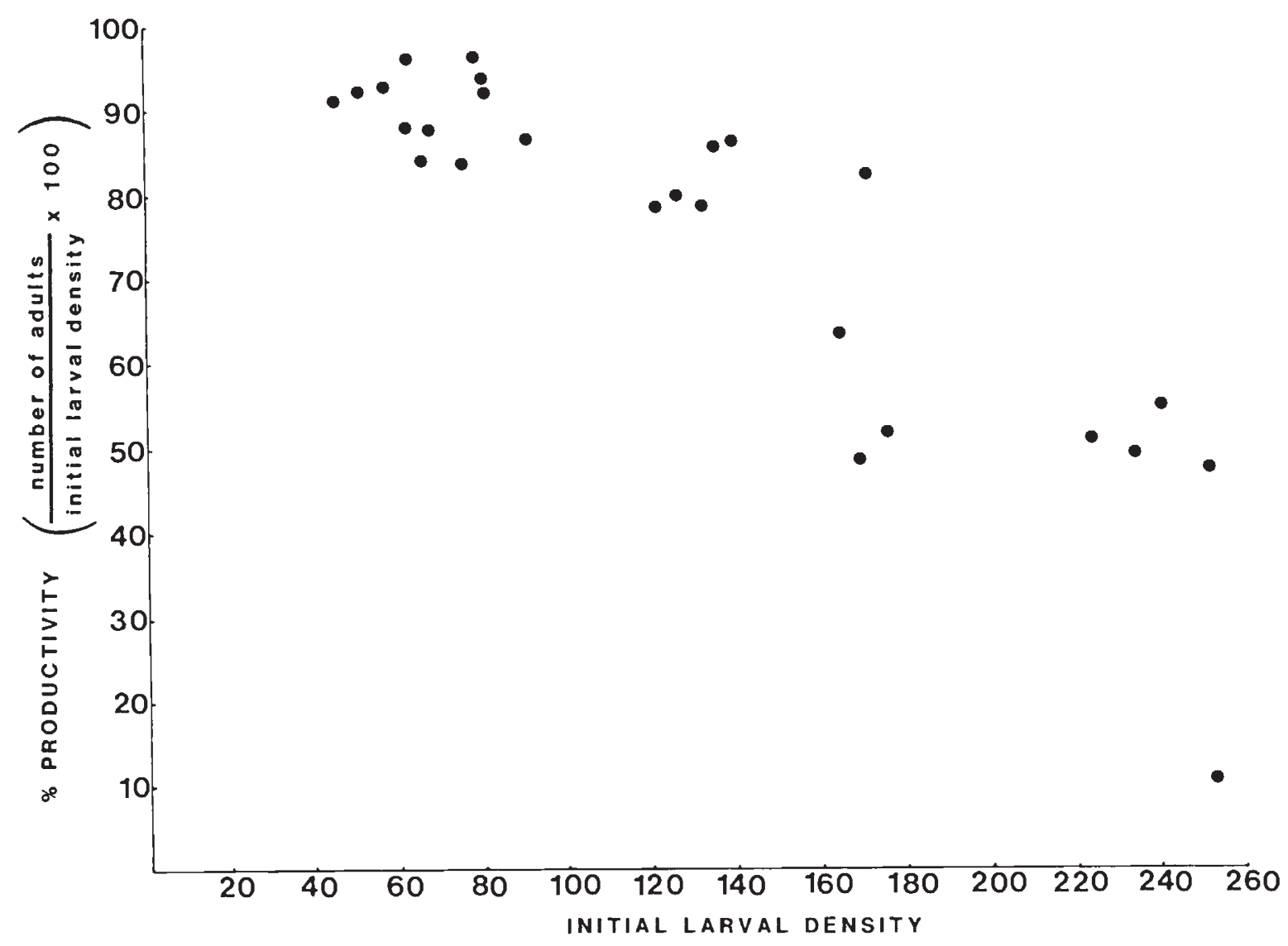

Figure 1. Productivity of vials for a range of initial larval densities.

Acknowledgements We thank the SERC for financial support, Dr A. Hoffman for the recipe for the spoons, Dr M. Bulmer for helpful discussions, Mrs D. Souter for technical assistance, Mrs J. Barbour for typing the manuscript and an anonymous referee for helpful comments.

\section{REFERENCES}

Battaglia, B. AND SMiTh, H. 1961. The Darwinian fitness of polymorphic and monomorphic populations of Drosophila pseudoobscura at $16^{\circ} \mathrm{C}$. Heredity, $16,475-484$.

BELL, G. 1982. The Masterpiece of Nature. Croom Hielm, London.

BOS, M. AND SCHARLOO, w. 1974. The effects of disruptive and stabilizing selection on body size in Drosophila melanogaster. III. Genetic analysis of two lines with different reactions to disruptive selection with mating of opposite extremes. Genetica, 45, 71-90.

BRITTNACHER, J. G. 1981. Genetic variation and genetic load due to the male reproductive component of fitness in Drosophila. Genetics, 97, 719-730.

CAligari, P. D. S. AND BABAN, D. F. A. 1981. The effect of parental age on sternopleural chaeta number in Drosophila melanogaster. Heredity, 47, 105-110.
ELLSTRAND, N. AND ANTONOVICS, J. 1985. Experimental studies of the evolutionary significance of sexual reproduction. II. A test of the density-dependent selection hypothesis. Evolution, 39, 657-666.

FOWLER, K. AND SEGER, J. 1983. Disruptive or directional selection? Nature, 304, 376.

KEARSEY, M. J. 1965. The interaction of food supply and competition in two lines of Drosophila melanogaster. Heredity, 20, 169-181.

KVELLAND, I. 1965. Some observations on the mating activity and fertility of Drosophila melanogaster males. Hereditas, 53, 281-306.

LEVENE, H. 1953. Genetic equilibrium when more than one ecological niche is available. Amer. Natur., 87, 131-133.

MAYNARD SMITH, J. 1971. What use is sex? J. theor. Biol., 30, 319-335.

PARTRIDGE, L., MACKAY, T. F. C. AND AITKEN, S. Male mating success and fertility in Drosophila melanogaster. Genet. Res. In Press.

PÉREZ-TOMÉ, J. M. AND TORO, M. A. 1982. Competition of similar and non-similar genotypes. Nature, 229, 153-154.

STEELE, R. H. 1984. An investigation of male mating success in Drosophila subobscura Ph.D. Thesis, University of Edinburgh.

WIlliaMS, G. C. 1975. Sex and Evolution. Princeton University Press, Princeton, New Jersey. 